\title{
Anterior cervical transvertebral approach for resection of an intraspinal ventral lesion: illustrative case
}

\author{
*Dongao Zhang, MD, ${ }^{1}$ Tao Fan, MD, PhD, ${ }^{1}$ Wayne Fan, ${ }^{2}$ and Xingang Zhao, MD ${ }^{1}$ \\ ${ }^{1}$ Spine Center, Sanbo Brain Hospital, Capital Medical University, Beijing, People's Republic of China; and ${ }^{2}$ Faculty of Science, The University of British Columbia, Vancouver, \\ British Columbia, Canada
}

\begin{abstract}
BACKGROUND The anterior cervical corpectomy and fusion approach has been reported for the removal of ventral cervical tumors. However, the normal cervical vertebral body and the adjacent intervertebral discs have to be sacrificed. In this paper, the authors describe a novel anterior cervical transvertebral approach for the resection of cervical intraspinal ventral lesions.

OBSERVATIONS A patient presented with an anteriorly placed extramedullary cyst. An anterior cervical transvertebral open-window and close-window approach was designed and applied to resect an intraspinal ventral enterogenous cyst. With this novel technique, a square was cut through the whole vertebral body at the four sides. After the cyst resection, the bone block was restored and fixed with a titanium miniplate. The lesion was totally resected, and the compression of the spinal cord was relieved. The physiological function of the cervical spine was kept intact after the operation. There was no postsurgical complication. The cervical alignment was normal at the 1-year postoperative follow-up.
\end{abstract}

LESSONS The anterior cervical transvertebral open-window and close-window approach was developed and confirmed to be effective for the resection of cervical intraspinal lesions. The cervical physiological structure and function can be restored with this new technique.

https://thejns.org/doi/abs/10.3171/CASE2190

KEYWORDS cervical spine; intraspinal lesion; ventral lesion; enterogenous cyst; surgical technique

Resection of ventral intradural spinal canal lesions may affect the spinal cord and adjacent intradural structures; thus, the selection of an appropriate approach and adequate exposure are of great importance for totally removing these lesions. Most ventral cervical spinal canal lesions are benign nerve sheath tumors, meningiomas, and enterogenous cysts. ${ }^{1}$ During the past 15 years, posterolateral approaches through hemilaminectomy have been common, and low-risk procedures are usually performed by spinal neurosurgeons. ${ }^{2,3}$ Sometimes, partial resection of the lateral bone, such as the facet joint and the vertebral pedicle, may facilitate exposure and resection. ${ }^{4}$ In many instances, these central ventral lesions may produce some rotation of the spinal cord, as well as lateral displacement, that can further facilitate their resection. ${ }^{5}$

There are particular circumstances in which dorsal access can be problematic, including calcification or tumors accompanied by significant pial involvement. $^{6,7}$ For example, ventral lesions located at a thoroughly ventral site with some degree of spinal cord compression, as well as prominently bilateral ventral tumor extension with no rotation or displacement of the spinal cord, can be difficult to safely resect via a posterolateral approach. Under these circumstances, direct visualization is indispensable for safe dissection to avoid damaging the delicate spinal cord vascular structure. To date, the anterior cervical corpectomy and fusion (ACCF) approach has been reported for the removal of ventral cervical tumors. ${ }^{8-10}$

However, the normal cervical vertebral body and the adjacent intervertebral discs have to be sacrificed when adopting the ACCF approach. Herein, we report a novel anterior cervical transvertebral (ACTV) open-window and close-window approach for the resection of ventral enterogenous cysts. Through this ACTV approach, the integrity of the vertebral body was restored, and the intervertebral discs were kept intact.

ABBREVIATIONS ACCF = anterior cervical corpectomy and fusion; ACTV = anterior cervical transvertebral; MRI = magnetic resonance imaging. INCLUDE WHEN CITING Published April 26, 2021; DOI: 10.3171/CASE2190.

SUBMITTED February 12, 2021. ACCEPTED February 19, 2021

* D.Z. and T.F. contributed equally to this work and share first authorship.

(C) 2021 The authors, CC BY-NC-ND 4.0 (http://creativecommons.org/licenses/by-nc-nd/4.0/). 


\section{Illustrative Case \\ Presentation}

A 40-year-old woman presented to our service with a 2-month history of neck pain and discomfort. Her physical examination revealed no muscle strength or sense disorder. Magnetic resonance imaging (MRI) of the spine showed an anteriorly placed central extramedullary cyst at the C5 level (Fig. 1). The spinal cord was compressed, and surgical treatment was considered. To directly visualize this ventrally and centrally located small lesion for safe dissection and to avoid damaging the delicate epipial vascular structure, a novel ACTV open-window and close-window approach was designed as follows. All procedures performed in the study involving a human participant were in accordance with the ethical standards of the institutional and/or national research committee. Informed consent was obtained from the participant included in the study.

\section{Surgical Procedures \\ Step 1}

Through a normal anterior cervical approach, the anterior surface of the C5 vertebral body was exposed, and the C4-5 and C5-6 disc spaces were kept intact (Fig. 2A).

\section{Step 2}

According to the size of the C5 vertebral body, a $1.0 \times 1.5-\mathrm{cm}$ square space was designed within the vertebral borders. With an ultrasound bone blade, the square was cut through the whole C5 vertebral body at the four sides. Meanwhile, the upper and lower vertebral endplates were kept intact. The bone block was removed integrally, and the posterior longitudinal ligament was excised (Fig. $2 \mathrm{~B}$ and $\mathrm{C}$ ).

\section{Step 3}

The middle line of the spinal dura was recognized and incised. Then, the dura was hung over bilaterally. The arachnoid membrane and spinal cord pia mater and the cyst were dissected and exposed (Fig. 2D and E).

\section{Step 4}

The cyst was carefully and totally resected. The adhesion of the cyst to the ventral spinal cord pia mater was sharply dissected. The

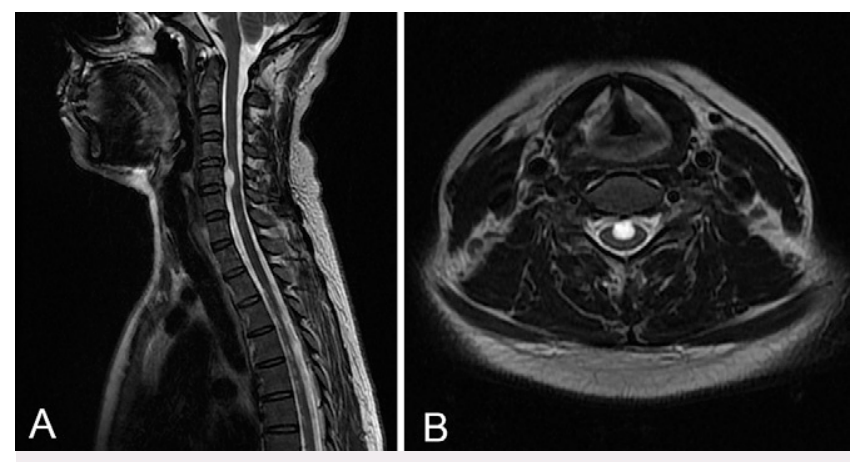

FIG. 1. Preoperative MRI of the spine shows an anteriorly placed central extramedullary cyst at the C5 level (A). The spinal cord was compressed by this ventrally located cyst (B).
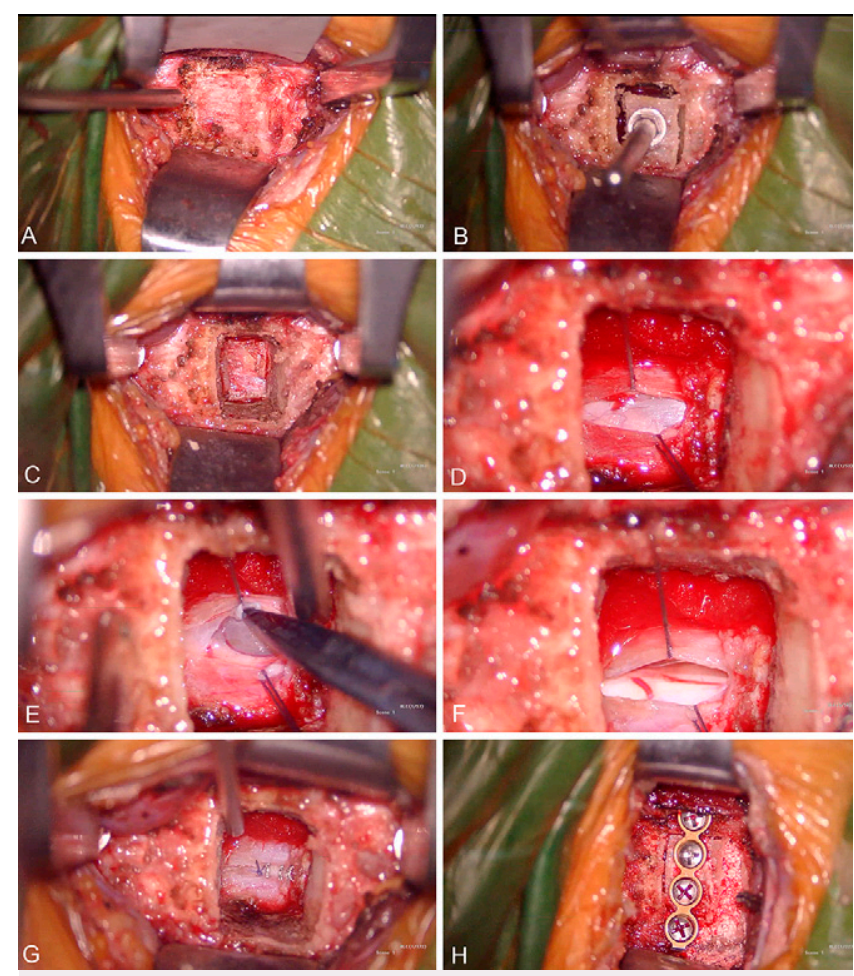

FIG. 2. The surgical procedures of the ACTV open-window and closewindow approach. A normal anterior approach was used to expose the anterior surface of C5 (A). A $1.0 \times 1.5-\mathrm{cm}$ square was cut through the whole $\mathrm{C} 5$ vertebral body at the four sides, and the bone block was removed integrally (B and $\mathbf{C})$. Spinal dural incision and tumor exposure ( $\mathbf{D}$ and $\mathbf{E})$. The cyst was resected, and the spinal cord vasculature was carefully protected $(\mathbf{F})$. The spinal dura was sutured in a watertight fashion via 5-0 surgical sutures and miniclips (G). The C5 bone block was restored and fixed with a titanium miniplate. The circumambient gap was padded by artificial bone substitute materials $(\mathbf{H})$.

frail spinal cord and the delicate epipial spinal cord vascular network were carefully protected (Fig. 2F).

\section{Step 5}

The spinal dura was sutured in a watertight fashion via 5-0 surgical sutures and miniclips (Fig. 2G).

\section{Step 6}

After saline flush, the $\mathrm{C} 5$ bone block was restored and fixed with a titanium miniplate. The circumambient gap was padded by artificial bone substitute materials. The surgical wound was closed routinely (Fig. 2H).

\section{Postoperative Follow-Up}

Postoperative MRI and computed tomography indicated that the cyst was totally resected. The compression of the spinal cord was relieved. The internal fixation was stable, and no height loss of the C5 vertebral body was observed (Fig. 3A-D). The patient had no postsurgical complications and was discharged from our hospital 6 

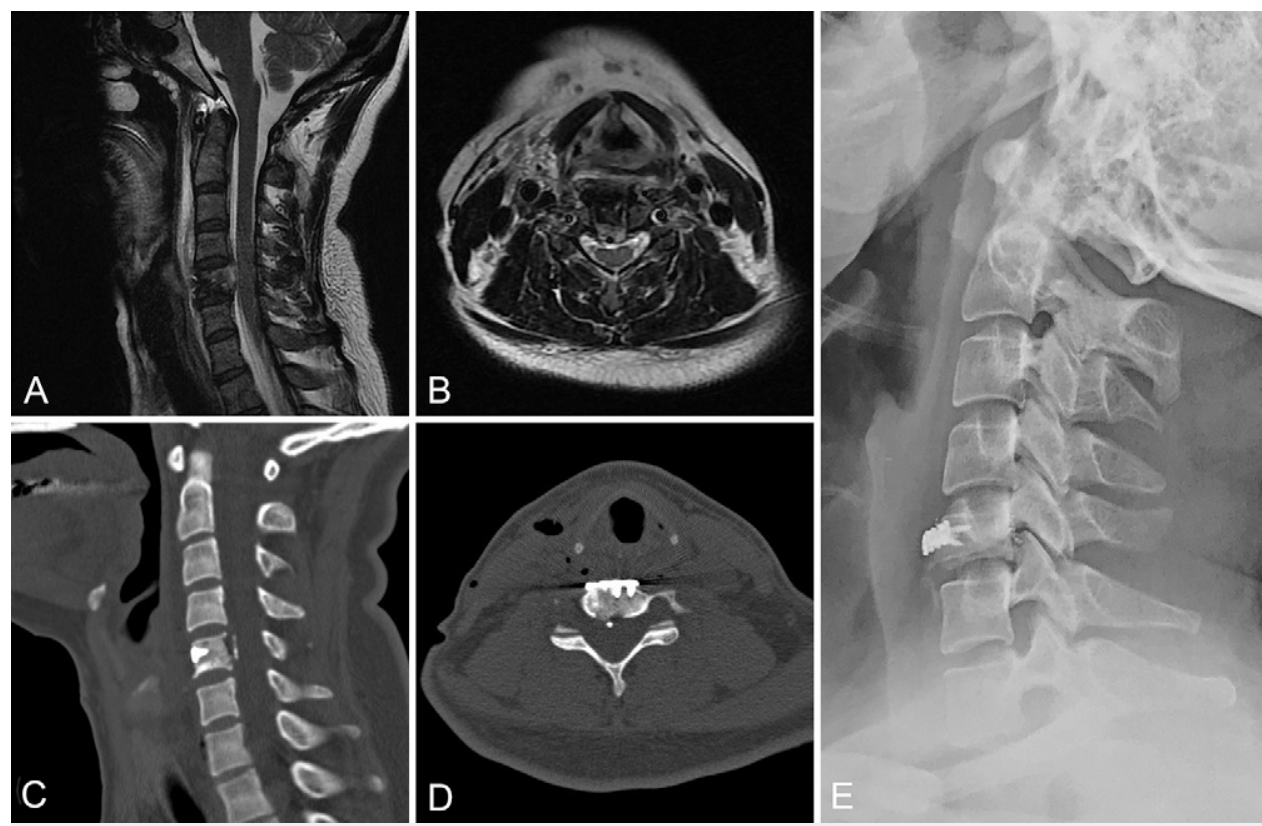

FIG. 3. Postoperative images. The cyst was totally resected with no compression of the spinal cord (A and B). The internal fixation was stable, and there was no height loss of the $\mathrm{C} 5$ vertebral body (C and D). The cervical alignment was normal at the 1-year postoperative follow-up (E).

days after the operation. A postoperative semi-rigid cervical collar was prescribed for 3 months. A radiograph showed normal cervical alignment at the 1-year postoperative follow-up (Fig. 3E).

\section{Discussion}

\section{Observations}

In the present case, an ACTV open-window and close-window approach was designed and applied to resect an intraspinal ventral enterogenous cyst. With this novel technique, the square was cut through the whole vertebral body at the four sides. After the cyst resection, the bone block was restored and fixed with a titanium miniplate. The lesion was totally resected, and the compression of the spinal cord was relieved. The physiological function of the cervical spine was kept intact after the operation. There was no postsurgical complication. The cervical alignment was normal at the 1-year postoperative follow-up. The cervical physiological structure and function were restored by this new technique.

\section{Lessons}

For most cervical intraspinal extramedullary tumors, a midline posterior lateral approach of hemilaminectomy is sufficient to expose and resect the tumor. However, for a ventrally located cervical intradural extramedullary tumor, the selection of a proper approach becomes more complicated. Compared with common posterior laminectomy and laminotomy approaches, a standard lateral hemilaminectomy approach can largely preserve the structure and mobility of the spine, but those approaches may also increase the chance of neurological impairment caused by traction of the spinal cord or nerve roots. ${ }^{11} A$ ventral mass located at the C3-7 level has greater challenges through a ventral approach. The cervical spinal canal becomes comparatively narrow at these levels. Meanwhile, the feature is accentuated at middle and low cervical levels because of the cervical cord enlargement. There is a greater risk of instability at these levels when partial facetectomy is needed to gain adequate ventral access. The ventral access through a posterolateral approach is further constrained because of the vertebral artery and the nerve roots. Root resection for exposure may cause significant complications, even with limited sacrifice of the dorsal root. In this circumstance, ventral exposure of this cervical level through a standard anterior cervical approach is necessary and standard.

An alternative ACCF approach has been described for resection of ventral extramedullary tumors. For example, O'Toole and McCormick reported a midline ventral intradural schwannoma of the cervical spinal cord causing myelopathy that was treated via ACCF. ${ }^{12}$ Soldozy et al. resected a giant granular cell tumor of the cervical spinal cord through a similar technique. ${ }^{11}$ Obviously, these ACCF approaches can directly access the ventral intradural spinal canal, but the vertebral bodies and the intervertebral discs have to be sacrificed. The reconstruction also leads to mobility loss of the cervical spine. Furthermore, anterior approaches tend to be longer and need more sustained retraction of the anterior cervical soft tissues than operations for degenerative diseases. This may increase the risk of postoperative complications, such as swallowing difficulty and voice deficits. Cerebrospinal fluid leakage, even an anterior cervical pseudomeningocele, can be another problem when performing anterior approaches for resection of cervical intradural lesions. Some authors have suggested that a spinal drain should be used for 3-5 days of continuous drainage. ${ }^{1}$ Currently, ACCF has been proved effective and still represents the mainstream alternative of an anterior cervical approach for resection of ventral intraspinal lesions.

In this case, our novel ACTV open-window and close-window approach was successfully used to access the ventrally and centrally located cyst, and the tumor was totally resected, meaning that the surgical disturbances of the delicate epipial spinal cord vascular 
network and the frail spinal cord were minimized as much as possible. By putting back the cut bone block and keeping the intervertebral discs intact, the integrity of $\mathrm{C5}$ and the alignment of the cervical spine were restored. The patient reported no postoperative complications, including neurological impairment or cerebrospinal fluid leakage. There was no mobility loss of the cervical spine. A radiograph showed normal cervical alignment at the 1-year follow-up. The ACTV open and close window approach was invented and verified to be effective for the resection of cervical intraspinal lesions, and the cervical physiological structure and function could be restored with this new technique. The limitation of the visual field and the exposure are obvious disadvantages of this pilot ACTV approach. The ACTV approach is applicable only to small and centrally located lesions. The indication and application of this pilot approach should be further explored.

\section{Acknowledgments}

This article was supported by the Beijing Municipal Science and Technology Commission (Z191100006619040, T.F.) and the Capital Health Research and Development of Special (2020-2-8011, T.F.).

\section{References}

1. Angevine PD, Kellner C, Haque RM, McCormick PC. Surgical management of ventral intradural spinal lesions. J Neurosurg Spine. 2011;15(1):28-37.

2. McCormick PC, Post KD, Stein BM. Intradural extramedullary tumors in adults. Neurosurg Clin N Am. 1990;1(3):591-608.

3. Steck JC, Dietze DD, Fessler RG. Posterolateral approach to intradural extramedullary thoracic tumors. J Neurosurg. 1994;81(2): 202-205.

4. Sridhar K, Ramamurthi R, Vasudevan MC, Ramamurthi B. Limited unilateral approach for extramedullary spinal tumours. $\mathrm{Br} J$ Neurosurg. 1998;12(5):430-433.

5. Martin NA, Khanna RK, Batzdorf U. Posterolateral cervical or thoracic approach with spinal cord rotation for vascular malformations or tumors of the ventrolateral spinal cord. $J$ Neurosurg. 1995;83(2):254-261.
6. Freidberg SR. Removal of an ossified ventral thoracic meningioma. Case report. J Neurosurg. 1972;37(6):728-730.

7. Gezen F, Kahraman S, Canakci Z, Bedük A. Review of 36 cases of spinal cord meningioma. Spine (Phila Pa 1976). 2000;25(6):727-731.

8. Muhammedrezai S, Ulu MO, Tanriöver N, et al. Cervical intradural ventral arachnoid cyst resected via anterior corpectomy with reconstruction: a case report. Turk Neurosurg. 2008;18(3):241-244

9. Iwasaki Y, Koyanagi I, Hida K, Abe H. Anterior approach to intramedullary hemangioblastoma: case report. Neurosurgery. 1999; 44(3):655-657.

10. Sohn MJ, Park HC, Park HS, et al. Anterior cervical corpectomy and fusion using miniplate and screws in a 7-year-old child with eosinophilic granuloma of the cervical spine. Spine (Phila Pa 1976). 2001;26(10):1193-1196.

11. Soldozy S, Syed HR, Jha RT, et al. Giant granular cell tumor of the cervical spinal cord resected via anterior corpectomy with reconstruction: technical note and review of literature. World Neurosurg. 2020;139:136-141.

12. O'Toole JE, McCormick PC. Midline ventral intradural schwannoma of the cervical spinal cord resected via anterior corpectomy with reconstruction: technical case report and review of the literature. Neurosurgery. 2003;52(6):1482-1486.

\section{Disclosures}

The authors report no conflict of interest concerning the materials or methods used in this study or the findings specified in this paper.

\section{Author Contributions}

Conception and design: T Fan. Acquisition of data: Zhang. Analysis and interpretation of data: Zhang. Drafting the article: Zhang, W Fan. Critically revising the article: T Fan, W Fan, Zhao. Reviewed submitted version of manuscript: T Fan, Zhang, Zhao. Approved the final version of the manuscript on behalf of all authors: T Fan. Administrative/ technical/material support: W Fan. Study supervision: T Fan.

\section{Correspondence}

Tao Fan: Capital Medical University, Beijing, People's Republic of China. fant@ccmu.edu.cn. 